\title{
From diagnosis to treatment: eagle syndrome
}

\begin{abstract}
Eagle syndrome (ES) is a symptom complex including recurrent neck and throat pain, foreign body sensation in the throat and dysphagia as a result of the elongation of the styloid process or the mineralization of the stylohyoid ligament complex. The symptoms of ES may worsen with lateral rotation of the head and it may less commonly present with tinnitus, weakness on the tongue, altered speech, intermittent loss of taste or dry mouth. If carotid compression is present, it may also present with episodes of fainting or stroke. An asymptomatic elongated styloid process alone does not meet the criteria for a diagnosis of ES. The pathogenesis of this syndrome is still debatable. It may be treated either nonsurgically or surgically. Pain modulators, transpharyngeal local infiltrations of anesthetic solutions or steroid medications, or neck exercises are among the non-surgical options. Surgical treatment includes intraoral or extraoral approaches, both of which comprise resection of the elongated styloid process.
\end{abstract}

Keywords: eagle syndrome, elongated styloid process, stylocarotid syndrome, pain
Volume 8 Issue 3 - 2017

\author{
Nelli Yıldırımyan,' Mustafa Daloğlu, ${ }^{2}$ Alper \\ Sindel,' Mehmet Ali Altay' \\ 'Department of Oral and Maxillofacial Surgery Faculty of \\ Dentistry Akdeniz University, Turkey \\ ${ }^{2}$ Clinic of Otolaryngology Antalya Ataturk State Hospital,Turkey
}

Correspondence: Nelli Yıldırımyan, Research Assistant, Department of Oral and Maxillofacial Surgery, Faculty of Dentistry, Akdeniz University, Dumlupinar Bulvari 07058 Kampüs, Antalya, Turkey, Tel +90-535-7486808,

Email nyildirimyan@gmail.com

Received: June 01, 2017 | Published: August 22, 2017
Abbreviations: ES, eagle syndrome; ESP, elongated styloid process; ICA, internal carotid artery; ECA, external carotid artery; OPTG, orthopantomography

\section{Introduction}

Eagle syndrome (ES) was originally described and named as "stylalgia" by Dr. Watt W. Eagle in 1937 to define a symptom complex including recurrent neck and throat pain, globus pharyngeus (foreign body sensation in the throat) and dysphagia as a result of the elongation of the styloid process or the mineralization of the stylohyoid ligament complex ${ }^{1,2}$ ES may be classified into two types: classic styloid syndrome and styloid-carotid artery syndrome (stylocarotid syndrome). ${ }^{3}$ It more commonly affects females and individuals in their third to fifth decades of life. ${ }^{4}$ The pathogenesis of this syndrome is still debatable. ${ }^{1}$ ES may be treated non-surgically or surgically depending on the severity of the symptoms. ${ }^{5}$

\section{Discussion}

Styloid process is a projection of the temporal bone, lying between the internal and external carotid arteries, anterior to the mastoid process and lateral to the tonsillar fossa where glossopharyngeal (IX), vagal (X) and hypoglossal (XII) nerves, internal carotid artery and internal jugular vein are located1. Facial nerve (VII) also runs anterior and medial to the styloid process. ${ }^{6}$

Physiological length of the styloid process varies between 20 to 25 millimeters and a process longer than 30 millimeters is defined as an elongated styloid process (ESP). ESP has an incidence of 4-28\% in the general population but only $4-10 \%$ of these individuals are symptomatic and therefore diagnosed with Eagle Syndrome6. Although periostitis, osteitis and reactive ossifying hyperplasia after surgery, trauma or local chronic irritation are considered in the etiology of this condition, the true cause of ES still remains to be elucidated. ${ }^{1,4}$

Cranial nerves V, VII, IX or X, internal (ICA) or external (ECA) carotid arteries or the jugular vein may be effected according to the length, width, angulation, direction, and the degree of calcification of the styloid process or the styloid ligament. ${ }^{4}$ Based on the involvement of the cranial nerves or the vascular structures, ES is sub-classified into classic styloid syndrome or the styloid-carotid artery syndrome, where the latter is associated with transient ischemic attacks as a result of pressure on the ICA or ECA. ${ }^{3,4}$

Symptoms of ES may include retroauricular and occipital pain, which worsens with lateral rotation of the head, dysphagia (difficulty during swallowing), odynophagia (pain during swallowing), tinnitus, tenderness during palpation at the retroauricular region, weakness on the tongue, altered speech, intermittent loss of taste, neck swelling, dry mouth, limited mouth opening or, if carotid compression is present, it may present with episodes of fainting or stroke. ${ }^{1,3-5}$ An ESP may remain asymptomatic as long as it does not impinge on adjacent structures. ${ }^{3}$ The length of the process and the severity of the symptoms do not show a correlation and patients with extremely long styloid processes may remain asymptomatic and unaware of their condition until routine radiographic examination. ${ }^{6}$

Orthopantomographic (OPTG), lateral-oblique mandibular or lateral head and neck radiographs may be helpful in the diagnosis of ES. If the initial radiographic assessment is done with lateral radiographs, it is important to obtain an additional anteroposterior radiograph, to monitor the patient for bilateral involvement. ${ }^{1}$ Conebeam computerized tomographic images may also be useful. ${ }^{4}$

An ESP may be observed in three distinct views on the OPTG images. Type I (Figure 1) reveals an uninterrupted ESP, type II (Figure 2) represents the styloid process being joined to the stylohyoid ligament by a single pseudo-articulation, and type III (Figure 3) consists of interrupted segments of the mineralized ligament, which appear like multiple pseudo-articulations within the ligament. ${ }^{7}$

Physical examination should include palpation of the anterior pillar region (lateral tonsillar fossa). Physicians may feel the ESP during careful intraoral palpation by placing the index finger in the tonsillar fossa and applying gentle pressure. In patients with ES, palpation should produce pain in this region or referred pain in the ear, face or the head. It should also be noted that it is not possible to palpate a normal-sized styloid process. ${ }^{7}$ The feeling of relief after a local anesthetic infiltration at the palpated area may aid in the diagnosis. ${ }^{2,4}$

Differential diagnosis of ES includes temporomandibular joint diseases, migraine or cluster type headaches, trigeminal, glossopharyngeal or sphenopalatine (Sluder's syndrome) neuralgias, chronic laryngopharyngeal reflux, chronic tonsillopharyngitis, 
hyoid bursitis, histamine cephalgia, esophageal diverticula, cervical vertebral arthritis and benign or malign neoplasms. ${ }^{4,7}$

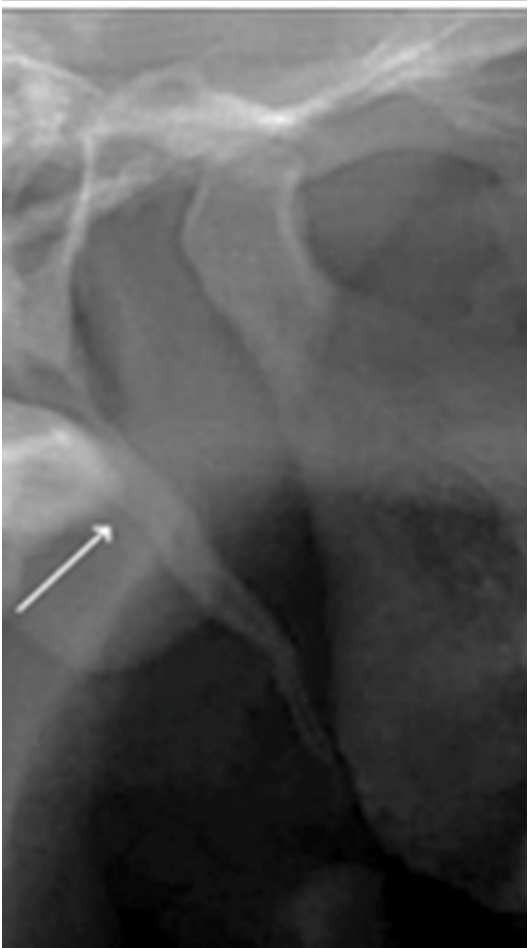

Figure I Type I, uninterrupted elongated styloid process (Radiograph from More CB \& Asrani MK [10]).

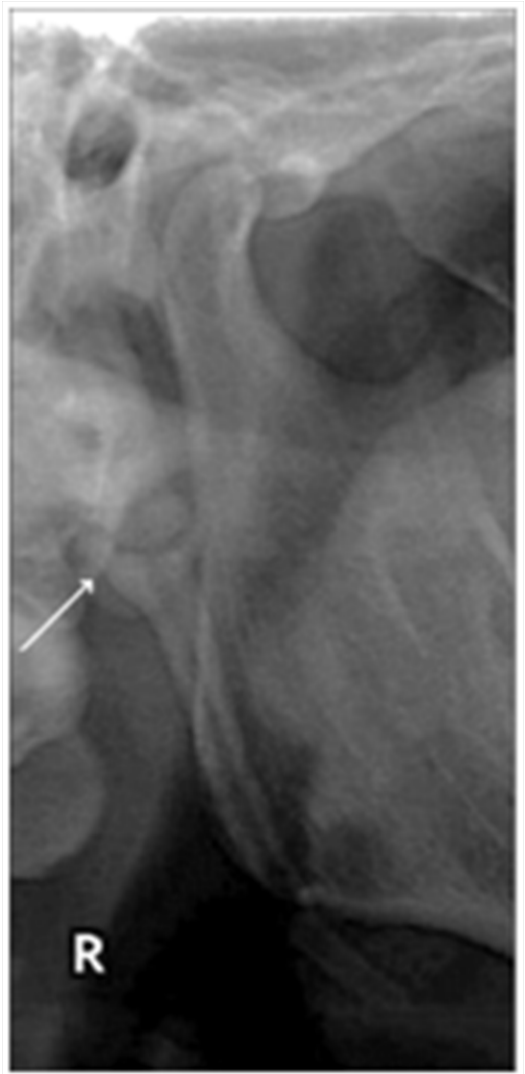

Figure 2 Type II, styloid process joining the stylohyoid ligament by a single pseudo-articulation (Radiograph from More CB \& Asrani MK [10]).

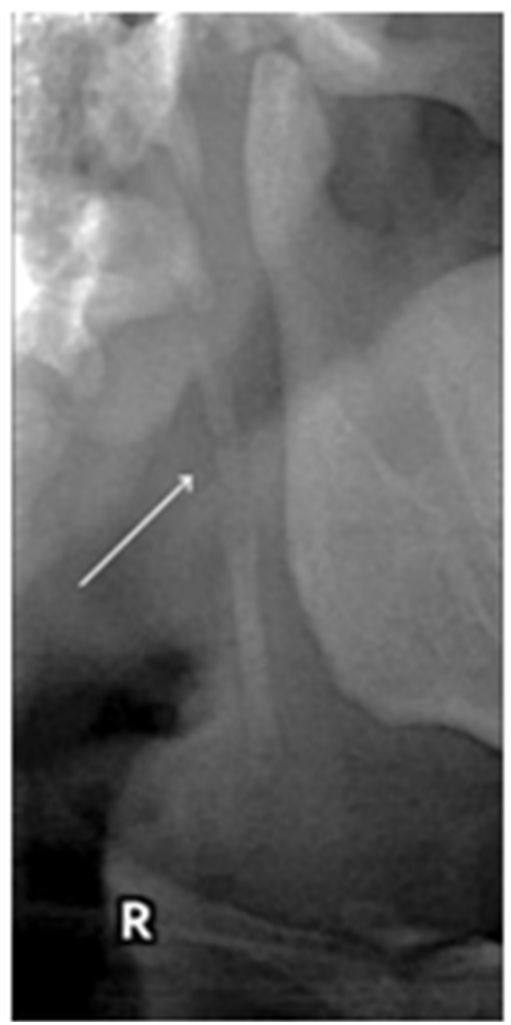

Figure 3 Type III, interrupted segments of the mineralized ligament looking like multiple pseudo-articulations (Radiograph from More CB \& Asrani MK [10]).

ES may be treated non-surgically or surgically. Non-surgical (conservative) treatment includes the use of pain modulators such as non-steroid anti-inflammatory drugs, pregabalin or gabapentin, transpharyngeal local infiltrations of anesthetic solutions or steroid medications into the tonsillar fossa, or neck exercises. ${ }^{1,4,5}$ Transpharyngeal manipulation involving manual fracturing of the styloid process is another documented approach although it carries a high risk of injury to the adjacent neurovascular structures 1. Despite the fact that non-surgical treatment options help reduce the pain, their effects last for a short period of time, particularly when the elongation or calcification is substantial. Therefore, surgery is considered the primary treatment modality in ES as it provides the most favorable outcome by eliminating the compressing (mechanical) factor in its entirety. ${ }^{4,5}$

Surgical treatment includes two main approaches, intraoral (transoral) or extraoral (transcervical). The intraoral approach requires less dissection, therefore the operation time is lesser and does not produce a cosmetic dissatisfaction. ${ }^{4,6}$ However the risk of inadequate resection is higher with this approach and, due to the non-sterile nature of the technique, it may give rise to deep cervical infections 2. The intraoral approach usually involves a tonsillectomy procedure as well. ${ }^{2}$

The extraoral approach provides a broad view and an aseptic access to the surgical target area and reduces the risk of major bleeding complications. However, operation lasts longer than the intraoral approach, and leaves an unaesthetic scar along the anterior border of the sternocleidomastoid muscle from the mastoid process to the hyoid bone. ${ }^{2,4,6}$ Several modifications to this conventional incision 
line were proposed by Buono et al., ${ }^{8}$ who used a face-lift incision typically performed during a parotidectomy and by Williams et al., ${ }^{9}$ who utilized a preauricular incision extending along the internal aspect of the tragus. It should, however, be noted that although these modifications provide more cosmetically acceptable post-operative results, they carry a greater risk of facial and greater auricular nerve injuries. ${ }^{2}$ Another extraoral approach utilizes the aid of an endoscope which places the skin incision near the retroauricular sulcus. ${ }^{2}$

Post-operative complications may include facial paresis, ear numbness, salivary fistula and trismus. ${ }^{2}$ Failures are reported to be as high as $20 \%$ in surgically treated ES patients because of the inadequate shortening of the styloid process or the development of subsequent fibrous entrapment syndrome, due to an intraoperative injury or entrapment within the fibrous tissue of the adjacent nerves. ${ }^{1}$

\section{Conclusion}

Eagle Syndrome is a symptomatic condition caused by the elongation of the styloid process or the mineralization of the styloid ligaments. ${ }^{1}$ ES should be considered as a differential diagnosis of pain-involving conditions of the occipital and retroauricular regions, particularly if it presents with dysphagia, foreign body sensation in the neck or an increase in the symptoms following the rotation of the head. ${ }^{5}$ A thorough clinical and radiological examination is of vital importance in diagnosis of the condition. Although there are both nonsurgical and surgical treatment alternatives, surgery is considered to be the primary treatment modality in patients with ES. ${ }^{4}$

\section{Acknowledgments}

Not applicable.

\section{Conflicts of interest}

Not applicable.

\section{Funding}

None.

\section{References}

1. Aldelaimi TN, Boskani SW, Ali SM, et al. Eagle Syndrome: An Unusual Cause Limited Mouth Opening and Surgical Management. J Craniofac Surg. 2017.

2. Chen R, Liang F, Han P, et al. Endoscope-Assisted Resection of Elongated Styloid Process Through a Retroauricular Incision: A Novel Surgical Approach to Eagle Syndrome. J Oral Maxillofac Surg. 2017;75(7):1442-1448.

3. Gupta A, Aggrawal A, Setia P. A rare fatality due to calcified stylohyoid ligament (Eagle syndrome). The Medico-legal journal. 2017;85(2):103-104.

4. Elimairi I, Baur DA, Altay MA, et al. Eagle's Syndrome. Head and Neck Pathology. 2015;9(4): 492-495.

5. Paiva ALC, Araujo JLV, Lovato RM, et al. Retroauricular pain caused by Eagle syndrome: A rare presentation due to compression by styloid process elongation. Revista da Associacao Medica Brasileira. 2017;(1922) 63(3):213-214.

6. Soylu E, Altan A, Sekerci AE, et al. An Asymptomatic and Overelongated Styloid Process. Case Rep Dent. 2017;2017:7971595.

7. Khandelwal S, Hada YS, Harsh A. Eagle's syndrome - A case report and review of the literature. The Saudi Dental Journal. 2011;23(4):211-215.

8. Buono U, Mangone GM, Michelotti A, et al. Surgical approach to the stylohyoid process in Eagle's syndrome. Journal of oral and maxillofacial surgery. 2005;63(5):714-716.

9. Williams JV, McKearney RM, Revington PJ. Eagle's syndrome: a novel surgical approach to the styloid process using a preauricular incision. Journal of oral and maxillofacial surgery. 2011;69(6):1617-1622.

10. More CB, Asrani MK. Evaluation of the styloid process on digital panoramic radiographs. Indian J Radiol Imaging. 2010;20(4):261-265. 